\title{
Economics of climate change: introducing the Basic Climate Economic (BCE) model
}

\author{
Lucas Bretschger, ${ }^{*}$ and Christos Karydas \\ ETH Zürich, Center of Economic Research, Zurich, Switzerland \\ ${ }^{*}$ Corresponding author. E-mail: lbretschger@ethz.ch
}

(Submitted 24 September 2018; revised 25 April 2019; accepted 27 April 2019; first published online 28 June 2019)

\begin{abstract}
Environmental economics models are often too complex to be communicated in an illustrative manner. For this reason, this paper develops the Basic Climate Economic (BCE) model that features core elements of macroeconomic and climate economic modelling, while allowing for an illustrative examination of the development path. The BCE model incorporates fossil stock depletion, pollution stock accumulation, endogenous growth, and climate-induced capital depreciation. We first use graphical analysis to show the effects of climate change and climate policy on economic development. Intuition for the different model mechanisms, the functional forms, and the effects of different climate policies is provided. We then show the model equations in mathematical terms to derive closed-form solutions and to run model simulations relating to the graphical part. Finally, we compare our setup to other models of climate economics.
\end{abstract}

Keywords: climate change; climate policy; endogenous growth; resource use; stock pollution

JEL classification: Q43; O47; Q56; O41

\section{Introduction}

Since the seminal contribution of Pigou (1920), it has been known that market failures caused by negative pollution externalities can be corrected by environmental policies. Climate change has been called the 'greatest market failure ever' (Stern, 2007). The method to derive policy conclusions thus appears to be standard; the mere fact that greenhouse gas emissions and their economic impacts are large should not have an impact on the basic concept. Yet, economic climate models and associated policy recommendations have suffered from different problems, notably the modelling of climate damages, the incomplete characterization of growth, or the lacking specifications of resource markets. Recently, this strand of research has even been harshly criticized (see Pindyck, 2013; Farmer et al., 2015; Stern, 2016). The reason for the critique lies in the difficulty in properly integrating climate change in economic models, in particular with respect to the interdependence between the ecological and the economic system, the 
long-run character of climate change, the link of emissions to natural resource depletion, and the nature and size of climate damages.

These issues pose serious challenges for developing a theory framework which includes sufficient precision to be useful while remaining clearly arranged to be intuitive. Specifically, one has to be careful when embedding ecological relationships related to climate change in an economic framework; model assumptions have to be in accordance with the results from natural sciences. ${ }^{1}$ Moreover, climate policy assessment models should reflect the state of the art in resource economics and dynamic macroeconomic modelling. As global warming affects the world economy for a very long time, economic development and its interactions with the resource stock in the ground and the pollution stock in the atmosphere are crucial and should be determined endogenously; a purely static analysis is not applicable in climate economics (Bretschger, 2017).

Recent papers have addressed important points of the critique by pushing the frontiers in economic theory, refining functional relationships and improving numerical calibration. ${ }^{2}$ But contributions have become very technical and quite specialized; for a broader audience it is often difficult to get an overview. The same holds true for quantitative models, for which Weitzman (2010) states: 'Because the climate change problem is so complex, there is frequent reliance on numerical computer simulations. These can be indispensable, but sometimes they do not provide a simple intuition for the processes they are modelling'. What is lacking in the literature is an illustrative general model showing the basic theoretical relationships of an economic climate model, including the most recent advances, in an intuitive manner. Such an approach can be used for educational activities and for communication, mainly within the scientific community but also with policy makers and the broader public. It can be especially useful in highlighting how different model assumptions affect the policy conclusions and how different policies are affecting the economy.

The present paper aims to fill this gap. We develop a simple flexible framework that integrates the economic approach to climate change labelled the 'Basic Climate Economic model'; henceforth the BCE model. In order to be useful for communication and broad knowledge diffusion, the paper starts by working with figures and verbal explanations. This should underline the basic reasoning in climate economics, show the different model parts in an intuitive form, and reveal the specific effects of different model assumptions. The model elements we are using concern natural resource stock depletion, pollution stock accumulation, pollution externalities in the form of climate damage functions, capital accumulation, and endogenous growth. Policies will affect one or multiple elements and have an effect on economic development. Also we will show the main differences between the BCE model and existing economic climate models that have drawn attention in the literature, namely the DICE model (Nordhaus, 2017) and the model of Golosov et al. (2014).

\footnotetext{
${ }^{1}$ Concerns have been raised about the formulation of the carbon cycle in the RICE/DICE model (e.g., Nordhaus, 2017) and the relevance of inertia in the climate system (see Dietz and Venmans, 2017).

${ }^{2}$ Uncertainty is included in (Lemoine and Traeger, 2014; Gerlagh and Liski, 2017; Bretschger and Vinogradova, 2018). Bretschger and Karydas (2018) study the effects of lags in the climate system on the social cost of carbon. Dietz and Stern (2015) show that optimal climate policy becomes significantly more stringent when the endogeneity of growth and the convexity of damages are included in the analysis and Bretschger and Pattakou (2019) explore the consequences of different damage functions. Gerlagh and Liski (2018) consider optimal carbon taxation when preferences deviate from the standard exponential type and there is no commitment to future climate policies.
} 


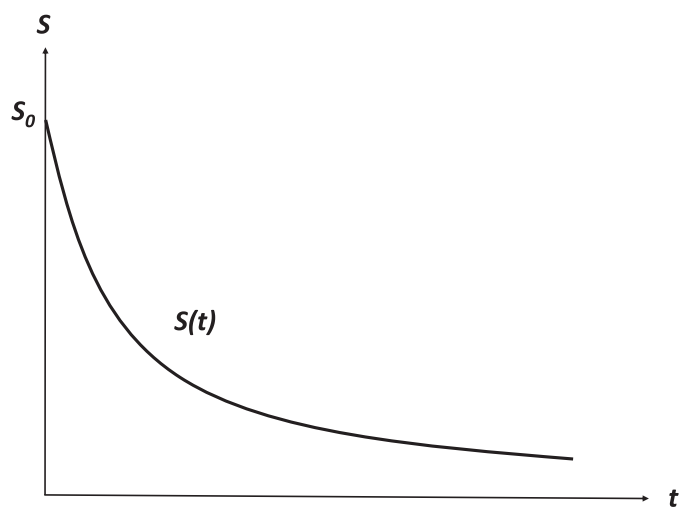

Figure 1. Resource stock depletion over time.

The remainder of the paper is organized as follows. Section 2 contains a graphical model analysis of the theory and of policy impacts. In section 3 we provide the theoretical foundation for the graphical approach, presenting analytical solutions and quantitative applications to replicate the figures of the previous section. Section 4 presents a comparison of our model to existing literature and section 5 concludes.

\section{Graphical approach}

This section develops the BCE model step by step, providing basic intuition about the different model mechanisms and their economic impact. Here we use curves and figures which will be mathematically derived in the next section of the paper. We start with a theory part and subsequently add policy effects.

\subsection{Climate economics theory}

The climate problem originates from the release of greenhouse gases into the atmosphere. The dominant share of these gases are carbon emissions which stem from burning fossil fuels. Stocks of these fuels are ultimately bounded so that an economic analysis should be based on the theory of optimal exhaustible resource depletion (Hotelling, 1931). For the sake of clarity we abstract here from new resource discoveries and extraction costs. ${ }^{3}$ When resources are continuously extracted, which we assume, the stock of remaining resources decreases over time. In figure 1 , resource stock $S$ starts at $S_{0}$ in time $t=0$ and decreases in time $t$ along the curved line.

The curvature of stock development as shown in figure 1 is based on the basic result of Hotelling (1931) which says that prices of exhaustible resources are driven by the resource rent, reflecting increasing resource scarcity over time. In standard resource models, the scarcity effect induces decreasing resource use over time so that the negative slope of the $S(t)$ function becomes smaller with growing $t$ (Dasgupta and Heal, 1974); see the next section for a more formal derivation. ${ }^{4}$

\footnotetext{
${ }^{3}$ These could be readily integrated following standard procedures of resource economics (e.g., Gaudet and Lasserre, 2015).

${ }^{4}$ If resource owners are not fully rational and/or forward-looking, the curve will have a different curvature but still has a negative slope which is sufficient to show the model effects graphically. Among others, the
} 


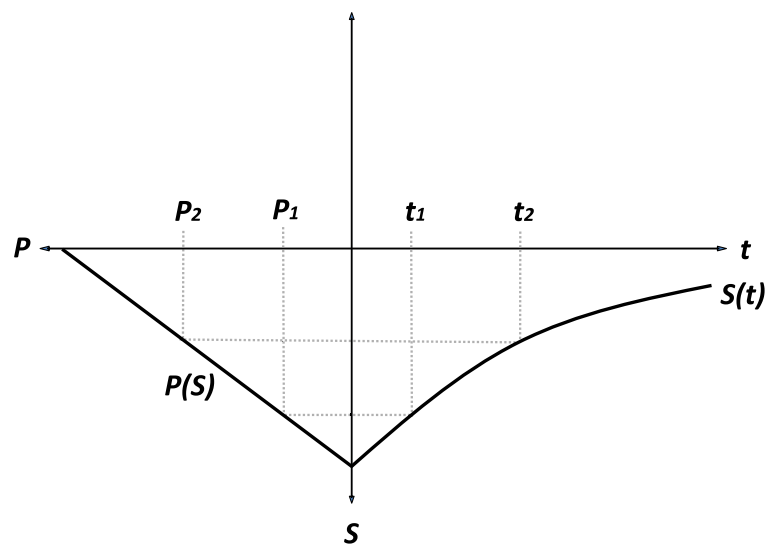

Figure 2. The relationship between pollution and resource stocks.

The stock of carbon in the atmosphere depends on total resource use in a linear way, with a fixed coefficient representing the carbon content of used fossil fuels. Natural decay of the pollution stock has been included in various economic models with a constant depreciation rate, which is convenient. In reality, however, the decay of greenhouse gases is a very complex and long-lasting process. Part of the emission stock disappears relatively quickly while the largest share stays in the atmosphere for several hundred years. ${ }^{5}$ Hence it is preferable to abstract from decay and to focus on a linear relationship between extracted resource stock $\left(S_{0}-S\right)$ and total pollution stock $P$. In figure 2 we have flipped the figure for resource stock of figure 1 around the horizontal axis and included pollution stock in the lower left quadrant measuring $P$ from right to left. The economy starts at $t=0$ and continuously depletes the resource stock which simultaneously raises pollution along the $P(S)$ line. Since $S$ is a function of time, this line implies the time evolution of pollution stock, i.e., $P_{t}=P\left(S_{t}\right)$. As shown in the figure, in times 1,2 the pollution stocks amount to $P_{1}$ and $P_{2}>P_{1}$; as the stock of resources gets depleted, pollution accumulates.

The next step concerns the impact of climate change, expressed in temperature, on the economy. Following recent climate physics, the relationship between pollution stock and temperature is almost linear (Hassler et al., 2016; Brock and Xepapadeas, 2017; Dietz and Venmans, 2017). ${ }^{6}$ Hence we do not need to introduce a separate variable for temperature but can directly proceed with the (appropriately scaled) pollution variable. The shape and the parameterization of the function relating pollution stock to economic damages are major points of concern and dispute; see Dietz and Stern (2015). To show the impacts in

depletion path will also be affected by policy as we will show below (see Gaudet (2007) for a discussion on ways in which the extraction path can be affected).

${ }^{5}$ See (IPCC, 2013, chapter 12) for more information on carbon concentration.

${ }^{6}$ Specifically, Dietz and Venmans (2017: 6), citing Matthews and Caldeira (2008) and others, state that 'the temperature response to a pulse emission of $\mathrm{CO}_{2}$ is approximately constant as a function of time, except for an initial period of adjustment that is very short, i.e., five to ten years' and (citing Matthews et al., 2009) that the warming effect of an emission of $\mathrm{CO}_{2}$ 'does not depend on the background concentration of $\mathrm{CO}_{2}$ in the atmosphere'. Conversely, other economics models have assumed growing effect of natural sinks (absorbing an increasing part of carbon emissions) and major delays in temperature response (e.g., van den Bijgaart et al., 2016) but we follow the most recent and accurate climate modelling here. 


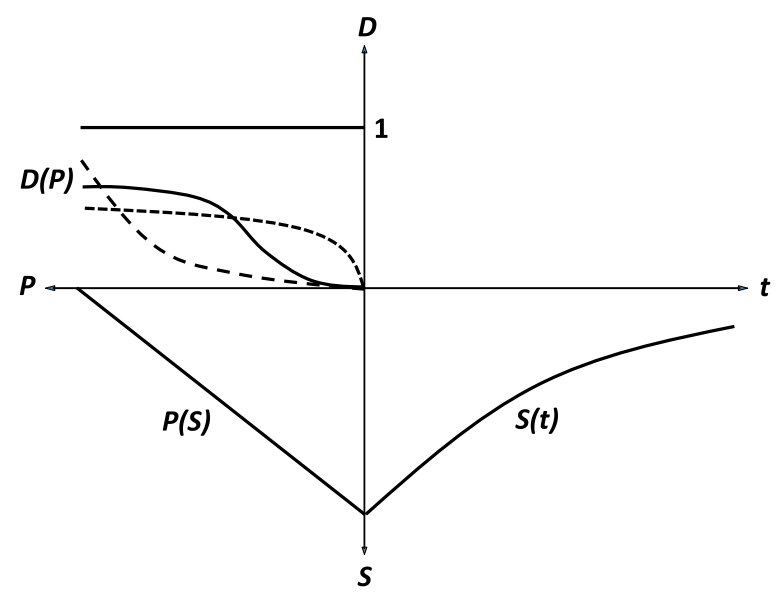

Figure 3. Different forms of the damage function $D(P)$.

the model, we need to further specify the kind of economic damages. Recent weather and climate disasters like hurricanes and landslides have harmed the affected regions most by destroying significant parts of the capital stocks, especially physical capital in the form of infrastructure, buildings, roads, etc. ${ }^{7}$ Correspondingly, in our model, part of the capital stock will be destroyed (i.e., depreciated) in each point in time due to climate change. Figure 3 shows the function for the damage rate $D$, expressed as a percentage of capital stock, and as a function of pollution stock $P$. The function is bounded between 0 and 1 and increasing in $P$; in principle it can be assumed convex, concave, or convex-concave (sigmoid), as shown in the figure. ${ }^{8}$

We are now ready to represent climate damages as a function of time in the first quadrant on the upper right; see figure 4 for the example of the convex-concave damage function. Each line linking the different functions translates the extracted resource stock to pollution and damages at a certain point in time. We see from the figure that the line in the first quadrant is shaped by the form of the damage function while its position depends on the size of available resource stock and pollution intensity of resource use.

To derive the impact of climate change on economic growth, climate-induced capital depreciation has to be confronted with the other dynamic elements stemming from capital accumulation. It is known from basic macroeconomics that the optimal consumption growth rate depends on the utility function of households, on marginal capital productivity, and on capital depreciation. The famous 'Keynes-Ramsey' rule widely used in growth theory says that consumption growth is positively affected by capital productivity, and negatively affected by discounting and the capital depreciation rate, which in our case is exacerbated by climate change. We can then represent the growth rate of consumption $(\hat{C})$ by the difference between the capital productivity effect, which we label $\Omega$,

\footnotetext{
${ }^{7}$ In our model, we use the terms 'capital' and 'physical capital' interchangeably. Both can be understood in the general sense to include productive tangible assets, such as buildings, machinery, infrastructure and renewable energy technologies, but also human capital.

${ }^{8}$ Note that a constant $D$ does not say that total damages $(D \cdot K)$ are linear in pollution stock $P$; in fact, total damages then grow with capital as a convex function of time while $P$ is concave in time so that total damages are a convex function of pollution stock, which is realistic.
} 


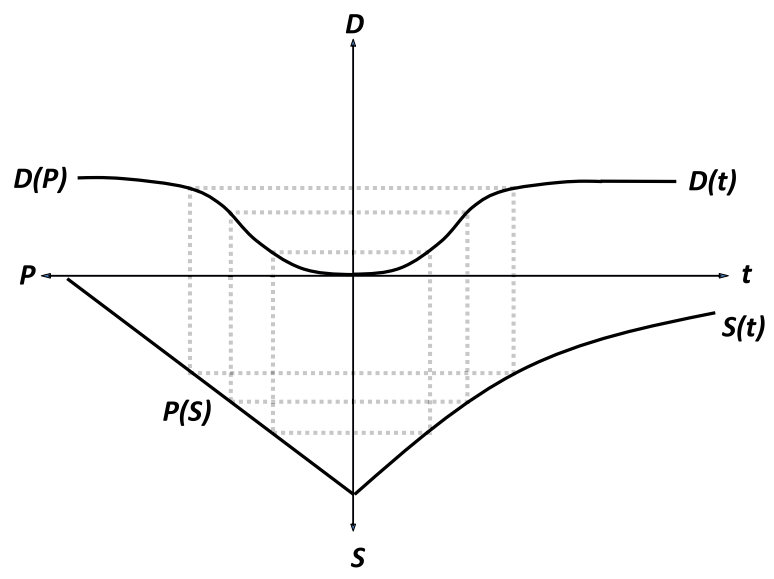

Figure 4. The convex-concave (sigmoid) damage function $D(P)$.

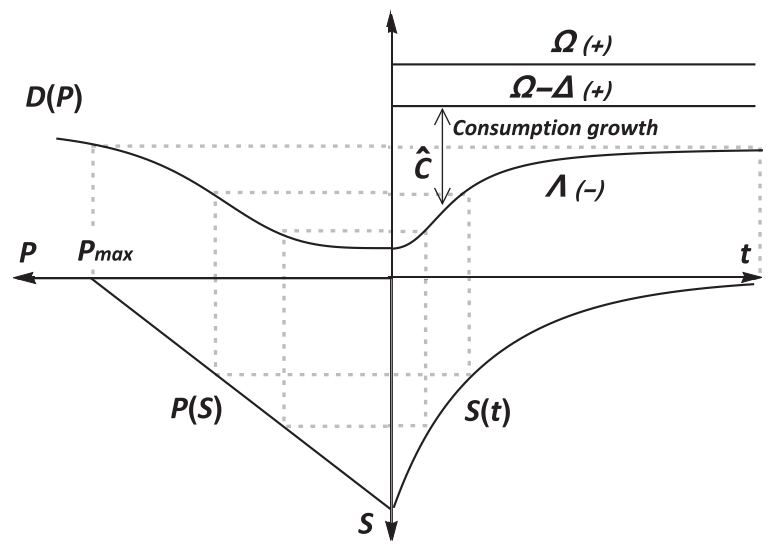

Figure 5. Effects of productivity $(\Omega)$, discounting $(\Delta)$, and depreciation $(\Lambda)$ on growth.

and the sum of the discounting effect $(\Delta)$, and the capital depreciation effect due to natural depreciation but also climate change $(\Lambda)$, i.e., growth is given by $\hat{C}=\Omega-\Delta-\Lambda$. As usual, we take the utility discount rate as given. Here we use our damage function to display the effect of climate-induced capital depreciation graphically.

Figure 5 shows the consumption growth rate for a convex-concave damage function as the difference between productivity net of discounting $(\Omega-\Delta)$ and climate-induced depreciation $(\Lambda)$. With given $\Omega$ and $\Delta$, it is immediately seen that the growth rate of the economy depends on capital damages $\Lambda$, and may be positive or negative depending on the model parameters. As pollution accumulates and climate deteriorates, so does economic growth, which reaches its steady state when pollution reaches its maximum level $\left(P_{\max }\right)$. The figure shows the case of a falling but positive consumption growth rate, which is likely for the world economy but may be unrealistic for a climate vulnerable 
(a)

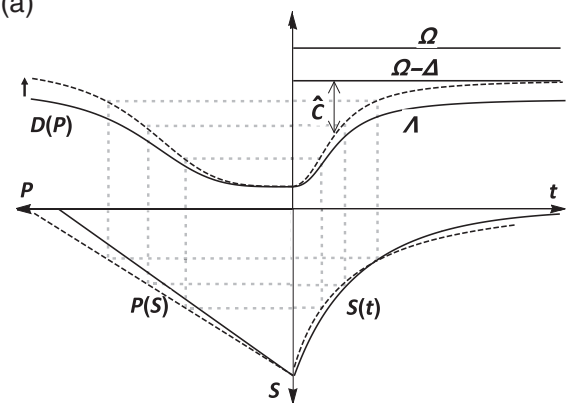

(c)

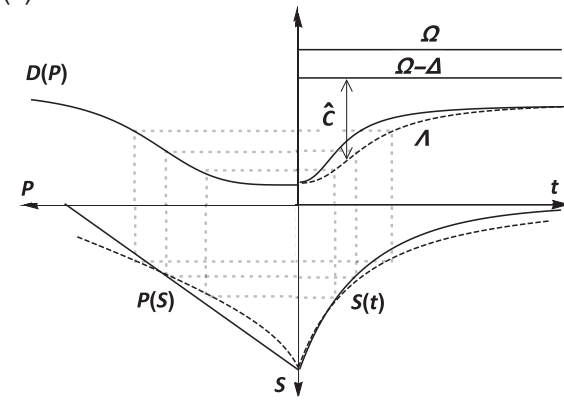

(b)

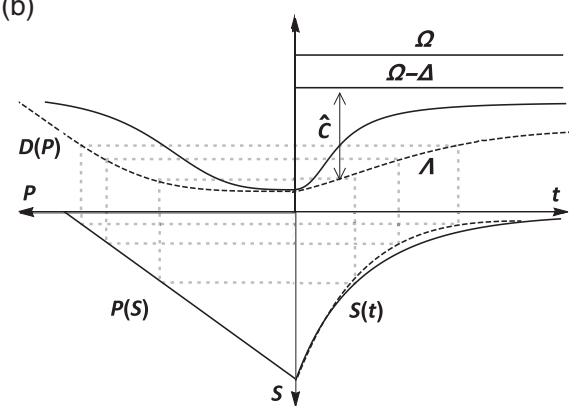

(d)

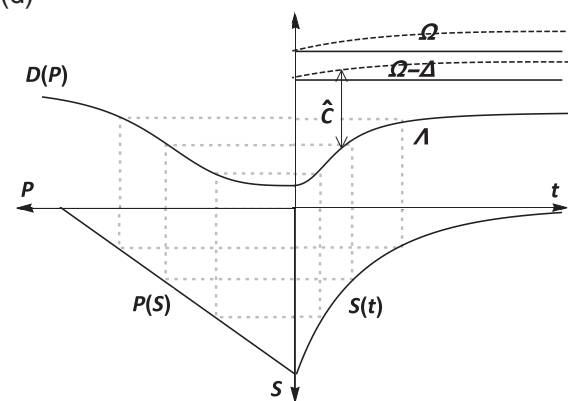

Figure 6. Changing model's parameters (solid - baseline model, dashed - new parameters). (a) Increasing pollution intensity (b) Convex damages (c) Lags in pollution accumulation (d) Increasing capital productivity.

region such as a small island state for which the $\Lambda$ line might cross the $\Omega-\Delta$ line from below, thus implying negative growth. ${ }^{9}$

\subsection{Changing modelling conditions}

The graphical model approach can now be used to discuss various core parts of the model and to show their impacts on economic development. Assuming the same shape of the damage function but a higher damage intensity of each pollution unit lowers the growth rate as shown in figure 6(a), where long-run growth becomes zero after a transition period; it may as well remain positive or become negative, this is a matter of appropriate calibration. The case of a purely convex damage function is shown in figure 6(b). In the example, the long-run growth rate remains positive due to decreasing resource use over time, but this is just one of the possible cases; the growth rate may also turn negative depending on the convexity of the damage function. ${ }^{10}$ The case of a delay in pollution accumulation is given in figure 6(c), where pollution has a relatively lower impact at the

\footnotetext{
${ }^{9}$ In the technical section that follows, we show that the BCE model features endogenous growth that results in constant capital productivity $\Omega$. Had we assumed that climate change affects the economy through the productivity channel, instead of the capital depreciation channel, $\Omega$ would decrease as pollution accumulated, while $\Lambda$ would be a constant, yielding a similar effect of pollution on economic development.

${ }^{10}$ Bretschger and Pattakou (2019) thoroughly examine the effects of convexity in pollution damage rates in the present framework.
} 
time of emission but an additional impact at a later stage because of the time lag between resource extraction and damages. ${ }^{11}$ Finally, changing capital productivity over time due to a sectoral change of the economy is represented in figure $6(d) .{ }^{12}$ In the favorable case of increasing capital productivity over time, as shown in the figure, economic growth is supported by structural change so that adverse climate effects can be alleviated; when sectoral change reduces capital productivity, both the productivity and the climate effect are harming the growth rate in the economy.

\subsection{Climate policies}

The graphical model approach can be conveniently used to show the effects of different climate policies. Real-life policy-making is rarely optimal and, therefore, the policies examined are not optimal policies, i.e. they do not necessarily align the decentralized equilibrium with the social planner solution. The most widely studied policy is the use of carbon taxes, the effects of which are shown in figure 7(a). Carbon taxation delays polluting resource extraction, and thus pollution accumulation. It follows that economic growth is higher all along the transition to the steady state, which is unaffected by the policy. ${ }^{13}$ It is an important feature of the model setup with costless extraction of exhaustible resources that taxation of resource use shifts the resource extraction profiles in time but never induces resource owners to leave resources unutilized in the ground. This would, however, exactly be needed for an effective climate policy, because climate physics predicts that the extraction of all fossil resources will cause very high damages, irrespective of the extraction profile; see Meinshausen et al. (2009) and McGlade and Ekins (2015). One way employed in the literature is the inclusion of increasing extraction costs and the costly development of clean backstop technologies as perfect substitutes to the incumbent resource, as in van der Ploeg and Withagen (2012). Here we take a different stand and examine the case of directly decommissioning part of the polluting resource stock as a policy option.

Figure 7(b) shows as an example the case of decommissioning part of the available stock of fossil fuels each year; the technical section shows how the policy is implemented in the model. With $S(t)$ we denote the available resource stock in time $t$, after the policy has been implemented, which naturally declines to zero over time. Variable $S(t)$ polluting reads as the effective stock of polluting resources, which is bounded by policy. The difference between these two curves is the amount of resources decommissioned up to time $t$. Total decommissioning is visualized by the red limitations to resource stock which is available for the economy. The stock measured as a difference between the red line and the origin is not available for commercial use and is thus not augmenting the pollution stock. Factor $\Pi$ shows the negative effect of policy on the growth rate of consumption: intuitively, since decommissioning reduces the profitability of fossil assets, this should be reflected in the rate of economic growth. In the end, if the benefit of reduced emissions (lower $\Lambda$ line) outweighs the cost of the policy (factor $\Pi$ ), economic growth is promoted.

\footnotetext{
${ }^{11}$ Bretschger and Karydas (2018) study the effects of lags in emissions diffusion in the present model.

${ }^{12}$ Bretschger and Smulders (2012) show the effects of structural change in a multisectoral model of endogenous growth with exhaustible resource extraction.

${ }^{13}$ The introduction or the increase of the carbon tax, however, has a negative level effect on income and consumption.
} 
(a)

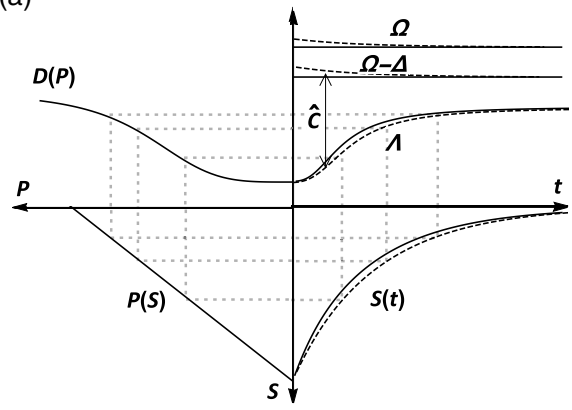

(c)

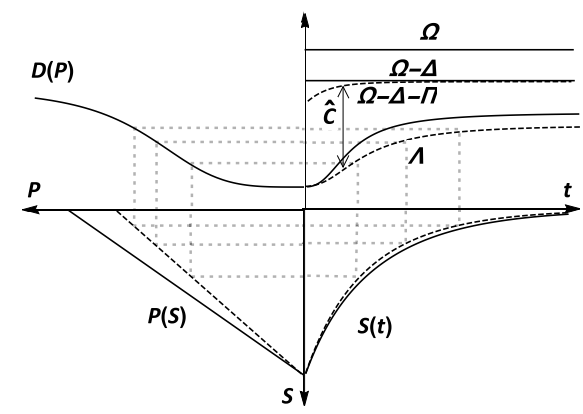

(b)

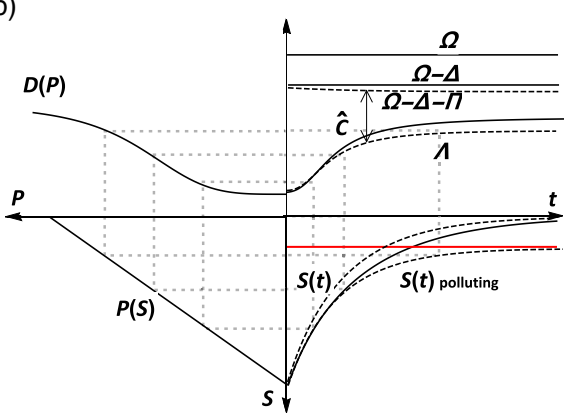

(d)

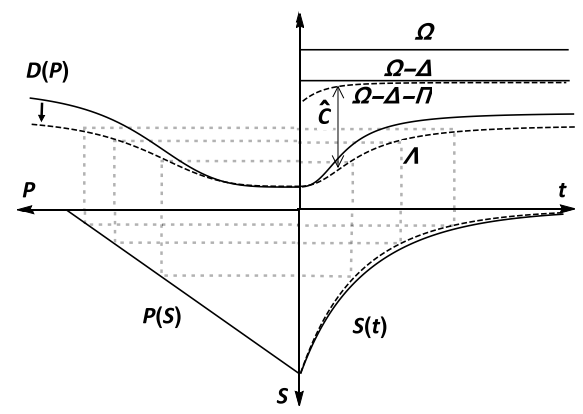

Figure 7. Effects of different policies (solid - baseline model, dashed - effects of policy).

(a) Carbon taxation (b) Decommissioning (c) CCS (d) Adaptation.

If a certain part of the capital stock is used for abatement activities, we obtain two effects in the model. First, because abatement is costly, we have to reduce capital productivity by a policy factor $\Pi$, which lowers the growth potential of the economy. For the second effect there are two cases. When each extracted resource unit has a lower impact on pollution stock $P$, as in the case of carbon capture and sequestration (CCS), the straight line in the lower left quadrant is rotated clockwise, as shown in figure 7(c). As a consequence, there is a lower (negative) impact of resource extraction on capital depreciation such that the growth rate is affected positively. When we look at adaptation to climate change, i.e., the building of dams or other specific protection, the pollution stock has a lower impact on capital depreciation as shown in the upper left quadrant of figure $7(\mathrm{~d})$, which again affects economic growth positively. The total effect of the policies is given by adding the two separate impacts.

Finally, it would be instructive to see how optimal policy would look like in our setup. The social planner solution would most closely resemble the case of resource taxation, where the tax rate is set at the social cost of carbon. ${ }^{14}$ Resource extraction would be

\footnotetext{
${ }^{14}$ Section 4 provides a comparison of our model to existing literature and calculates the social cost of carbon, i.e., the optimal carbon tax.
} 
stretched further in time (flatter extraction profile) and so would pollution accumulation. This would also induce a flatter growth profile towards the steady state, i.e., higher growth at all times during transition in comparison to the no-policy case.

\section{Formal analysis}

This section formally presents the theoretical foundation of the BCE model. The model builds on the two-sector AK model of endogenous growth introduced by Rebelo (1991), as modified by Bretschger and Karydas (2018) to include polluting non-renewable resources as a productive input, and pollution-induced damages to the capital stock. ${ }^{15}$ In comparison to the aforementioned contribution, here we abstract from lags in pollution accumulation, we include a more rigorous representation of the damage function (sigmoid instead of linear), and we examine a much larger set of climate policy alternatives. We will first present the basic model and subsequently the alterations needed to get the results for each policy option. Our analysis focuses on a closed economy in continuous time.

\subsection{Theory}

\section{Climate change and damages}

Before we describe the macroeconomic environment, we present our assumptions on the climate system, on pollution, and how it feeds back in the economy by destroying stocks of available capital. Polluting non-renewable resources are used as inputs in production. Let $S_{t}$ denote the stock of non-renewable resources available in time $t$ and $R_{t}$ the resource extraction. Extracting and burning fossil fuels in time $t$ depletes the resource stock and simultaneously adds to the existing stock of pollution $P_{t}$ according to:

$$
\dot{S}_{t}=-R_{t}, \quad \text { given } \quad S_{0}>0,
$$

and

$$
\dot{P}_{t}=\phi R_{t}, \quad \text { given } P_{0}>0,
$$

with $\phi>0$ the pollution intensity of the non-renewable resource. Resource extraction is decreasing over time leading to a decreasing time profile for the resource stock as in figure 1. Combining the above two equations leads to a linear relationship between the stock of pollution and the stock of non-renewable resources; the $P(S)$ line in figure $2:{ }^{16}$

$$
P_{t}=P_{0}+\phi\left(S_{0}-S_{t}\right) \text {. }
$$

As the stock of non-renewable resources gets depleted, pollution increases. When the whole stock is depleted, pollution gets its maximum value $P_{\max }=P_{0}+\phi S_{0}$. We

\footnotetext{
${ }^{15}$ Many endogenous growth models exhibit scale effects, with the counterfactual prediction that a growing population should have translated to higher economic growth; see Jones (1995). The two-sector AK model of Rebelo (1991) has no scale effects of this sort, while it comports with empirical macroeconomic evidence, most notably with: i) a declining price of equipment investment relative to the price of consumer nondurables; ii) a constant (nominal) savings rate; and iii) an increasing ratio of real investment to real GDP (see Felbermayr and Licandro, 2005).

${ }^{16}$ In the subsequent case of gradual decommissioning of the stock of polluting non-renewable resources as a policy option, the equivalent equation will read as $P_{t}=P_{0}+\phi\left(S_{0}-S_{t}\right)-\phi \int_{0}^{t} x_{s} S_{s} d s$, with $x_{t} \in(0,1)$ the expropriation rate at time $t$. Accordingly, both examined cases allow for a linear relationship between $P$ and $S$.
} 
use pollution stock as a measure of climate change. The linear relationship of equation (2), between the change in the variable responsible for climate change and greenhouse gas emissions, is well founded in the literature. ${ }^{17}$ Natural disasters caused by man-made pollution are increasingly harming economic activity by destroying available stocks of capital. In our model, climate change damages are measured as a percentage of the available stock of capital in each period. We will assume that pollution feeds back in the economy through a sigmoid damage function $D(P)$, according to:

$$
D\left(P_{t}\right)=\delta_{0}+\delta_{1}\left(1-\frac{1}{1+\delta_{2}\left(P_{t}-P_{0}\right)^{\eta}}\right),
$$

with $\delta_{0} \in[0,1]$, the natural depreciation of the capital stock, $\delta_{1} \in\left[0,1-\delta_{0}\right], \delta_{2}>0$, scaling parameters, and $\eta \geq 1$ a convexity parameter. A similar functional form is used in the latest DICE-2016R model but there damages reduce aggregate productivity and not capital stock; see Nordhaus (2017). With this damage function we make sure that damages as a percentage of the stock of capital are bounded between 0 and 1 , while at the same time we can calibrate it such that the mapping between pollution and damages is convex for the relevant range of available polluting resources, as typically advocated in the literature (e.g., Golosov et al., 2014). ${ }^{18}$

\section{Macroeconomic environment}

There are two financial assets owned by households: a stock of polluting non-renewable resources $S$, and capital $K$. There are also two economic sectors: the manufacturing sector that produces goods readily available for consumption, and the corporate sector that provides goods and services for investments that augment the stock of capital.

In order to produce the consumption good $Y$, the manufacturing sector combines a part of physical capital $K_{Y}$ with non-renewable resources $R$ in a Cobb-Douglas fashion:

$$
Y_{t}=A K_{Y t}^{\alpha} R_{t}^{1-\alpha}
$$

Parameters $\alpha \in[0,1]$ and $A>0$ represent the capital expenditure share and the productivity of the manufacturing sector, respectively. According to the Cobb-Douglas specification of (5), we assume that polluting non-renewable resources are essential inputs in the production of the consumer good. In fact, empirical evidence is inconclusive about whether the elasticity of substitution between capital and polluting energy resources is above unity or not. Van der Werf (2008) uses industry-level data from 12 OECD countries and consistently finds elasticities of substitution between energy and other inputs which are smaller than unity. Hassler et al. (2015) suggest very low elasticities of substitution between capital and energy in the short run, but high elasticities in the long run, while Papageorgiou et al. (2017) find elasticities above unity.

\footnotetext{
${ }^{17}$ See Hassler et al. (2016), Brock and Xepapadeas (2017) and Dietz and Venmans (2017).

${ }^{18}$ Choosing another damage function, bounded in $[0,1]$, from the ones presented in figure 3 would not alter the main results regarding the effects of different policies on economic development. A linear relationship $D(P)$ is commonly used in the literature for its analytical convenience (e.g., Grimaud and Rouge, 2014; Bretschger and Karydas, 2018). Analytical approximations of the social cost of carbon (SCC) using a complex damage structure similar to (4) have been provided by van den Bijgaart et al. (2016). For the relevant range of the available polluting resources, Golosov et al. (2014) approximate damages in GDP by an exponential function.
} 
Before we draw, however, any pessimistic conclusions about future economic development, note that in our framework there are two ways of substituting towards clean resources and cleaning up the pollution from the dirty resources at finite cost. First, capital $K$ is clean and is assumed to include renewable energy capital such as solar panels, dams and windmills; the availability of renewable energies clearly requires long-run capital investments. Second, we explicitly analyze the policy of cleaning up by looking at Carbon Capture and Sequestration (CCS) as already presented in the graphical part of the paper. With CCS we have directly the possibility to clean up the pollution stock at finite costs.

In turn, the corporate sector, responsible for providing the investment good $I$, has a linear technology in physical capital $K_{I}$ :

$$
I_{t}=B K_{I t}
$$

with $B>0$ a productivity parameter. The pollution stock is responsible for climate change and damages to the existing stock of capital through function $D(P)$ defined in $(4) .{ }^{19}$ Capital accumulation reads:

$$
\dot{K}=I_{t}-D\left(P_{t}\right) K_{t} \quad \text { given } \quad K_{0}>0 .
$$

Finally, households receive rents from physical capital and non-renewable resources and balance their income with expenditure on the consumption good $C$, and on the investment good $H$, the latter being the equivalent of savings in the present economy. In equilibrium, households demand the total consumption and investment goods, i.e., $C=Y$ and $H=I$ respectively, while capital is exactly shared between the manufacturing and the investment sector, i.e., $K_{Y}+K_{I}=K$; GDP in this economy reads $p_{Y} Y+p_{I} I$, with $p_{Y}, p_{I}$ the prices of the consumption and the investment good, respectively. Let us now proceed by describing the market economy and the equilibrium.

\section{Firms}

Let the consumption good $Y$ be the numeraire, i.e., $p_{Y}=1$. Firms in both sectors maximize instantaneous profits according to:

$$
\max _{K_{Y}, R}\left\{Y_{t}-p_{K t} K_{Y t}-p_{R t} R_{t}\right\} \text { and } \max _{K_{I}}\left\{p_{I t} I_{t}-p_{K t} K_{I t}\right\}
$$

with $p_{K}$ the rental price of capital, $p_{R}$ the price of non-renewable resources, and $p_{I}$ the price of the investment good, i.e., the price of investment in new forms of capital. Let us define with $\epsilon \equiv K_{Y} / K$ the share of total available capital employed by the manufacturing sector. With (5) and (6), this maximization gives the demand curves for non-renewable

\footnotetext{
${ }^{19}$ In order to capture any growth effects of climate change in a meaningful manner, one has two options. First, we could place damages directly on the production of the good responsible for investment. This would be final output when investment and consumption come from the same good, as usually done in the literature (e.g., Golosov et al., 2014) or the investment good in our case. With this modelling assumption, economic development would be affected through the productivity channel, i.e., factor $\Omega$ in figure 5 , which would decrease as pollution accumulates. Second, and equivalently, we can place damages directly on capital depreciation as advocated by Stern (2007). Here we follow the second approach.
} 
resources and capital in the two sectors:

$$
(1-\alpha) \frac{Y_{t}}{R_{t}}=p_{R t}, \quad \alpha \frac{Y_{t}}{\epsilon_{t} K_{t}}=p_{K t}, \quad p_{I t} B=p_{K t} .
$$

The last two equations imply a no-arbitrage condition for the use of capital between sectors: employing the marginal unit of capital in the two sectors should yield the same return.

\section{Households}

Households allocate their rental income from physical capital and non-renewable resources between expenditure on consumption $C$ and on additional capital formation $H$. Let $T$ represent generic non-distorting lump-sum transfers. Then, the income balance reads:

$$
p_{K t} K_{t}+p_{R t} R_{t}+T_{t}=C_{t}+p_{I t} H_{t} .
$$

Expenditure on capital formation adds to the existing stock of capital according to:

$$
\dot{K}=H_{t}-D\left(P_{t}\right) K_{t},
$$

although agents do not internalize damages to capital accumulation through higher levels of pollution. Total wealth reads $W=p_{I} K+p_{R} S$. Time-differentiation of total wealth using (1), (10), (11), and the fact that $p_{K}=p_{I} B$ from (9), leads to the household's dynamic budget constraint, according to:

$$
\dot{W}_{t}=\theta_{t} W_{t} \hat{p}_{R t}+\left(1-\theta_{t}\right) W_{t}\left(\hat{p}_{I t}+B-D\left(P_{t}\right)\right)-C_{t}+T_{t},
$$

with $\theta \equiv p_{R} S / W$, the share of the individual's resource wealth in the total assets; hats denote growth rates, i.e., $\hat{p}=\dot{p} / p$. Finally, the representative household chooses the time path of consumption $C$ and asset allocation $\theta$ in order to maximize lifetime utility:

$$
\int_{0}^{\infty} U\left(C_{t}\right) e^{-\rho t} d t
$$

subject to the budget constraint (12); $\rho>0$ is the intergenerational discount rate. We will assume throughout that households have constant relative risk aversion (CRRA) preferences according to $U(C)=C^{1-\sigma} /(1-\sigma)$, with $\sigma>0$ the inverse of the elasticity of intertemporal substitution. Following the empirical literature, we will focus on the case of $\sigma>1$. With $r$ being the aggregate rate of interest, the household optimization yields: ${ }^{20}$

$$
\begin{aligned}
\hat{C}_{t} & =\frac{1}{\sigma}\left(r_{t}-\rho\right), \\
\hat{p}_{R t} & =r_{t}=\hat{p}_{I t}+B-D\left(P_{t}\right) .
\end{aligned}
$$

Equation (14) is the usual Keynes-Ramsey condition for consumption growth. Equation (15) is a no-arbitrage condition between assets: accounting for depreciation,

\footnotetext{
${ }^{20}$ Households choose $C$ and $\theta$ in order to maximize (13) subject to (12). The corresponding Hamiltonian reads $\mathcal{H}_{t}=C_{t}^{1-\sigma} /(1-\sigma)+\lambda_{t}\left(\theta_{t} W_{t} \hat{p}_{R t}+\left(1-\theta_{t}\right) W_{t}\left(\hat{p}_{I t}+B-D\left(P_{t}\right)\right)-C_{t}+T_{t}\right)$, with $\lambda_{t}$ the shadow price of wealth. This optimization leads to equations $\lambda_{t}=C_{t}^{-\sigma}$ and $\hat{p}_{R t}=\hat{p}_{I t}+B-D\left(P_{t}\right)$, and to the costate equation $\lambda_{t}\left(\theta_{t} \hat{p}_{R t}+\left(1-\theta_{t}\right)\left(\hat{p}_{I t}+B-D\left(P_{t}\right)\right)\right)=\rho \lambda_{t}-\dot{\lambda}_{t}$.
} 
each asset should yield the same marginal return in equilibrium. In this closed economy, this return is the risk-free rate of interest $r$. Note that the first equation of (15) is the Hotelling rule for the price evolution of the non-renewable resource: the appreciation in the resource's marginal profitability - the resource price when no extraction costs are considered - should yield indifference between investing the rents of immediate extraction at a risk-free return $r$, or extraction next period at a price grown by the same rate. Finally, the above optimization must be augmented by the appropriate transversality condition, which reads:

$$
\lim _{t \rightarrow \infty} \lambda_{t} W_{t} e^{-\rho t}=0,
$$

with variable $\lambda=C^{-\sigma}$, the shadow price of total wealth. ${ }^{21}$

\section{Equilibrium}

In equilibrium, total demand for consumption and investment goods should equal their total supply, i.e., $C=Y$ and $H=I$. Given positive $K_{0}, S_{0}$, non-negative $P_{0}$, the dynamics of resource depletion, of pollution, and of capital accumulation (i.e., (1), (2) and (7)), along with the first order conditions for firms and households (i.e., equations (9), (14), (15)) and the transversality condition (16), completely characterize the dynamic behavior of the decentralized economy.

\section{Solving the basic model}

Let $u \equiv R / S$ be the resource depletion rate. Log-differentiating equations in (9) using $\hat{R}=\hat{u}-u$ from (1) and the definition of $u$, and (7) with $I=B(1-\epsilon) K$, leads to:

$$
\begin{aligned}
& \hat{u}_{t}=u_{t}-(\sigma-1) \hat{C}_{t}-\rho, \\
& \hat{\epsilon}_{t}=B \epsilon_{t}-(\sigma-1) \hat{C}_{t}-\rho .
\end{aligned}
$$

Finally, log-differentiating the production function (5) for $C=Y$ in equilibrium, using (7) with $I=B(1-\epsilon) K$ as before, and $\hat{\epsilon}$ and $\hat{u}$ from above, gives the time evolution of the consumption growth rate according to:

$$
\hat{C}_{t}=\underbrace{\frac{\alpha B}{\sigma}}_{\Omega \text {-productivity }}-\underbrace{\frac{\rho}{\sigma}}_{\Delta \text {-discounting }}-\underbrace{\frac{\alpha D\left(P_{t}\right)}{\sigma}}_{\Lambda \text {-depreciation }} .
$$

Expression (19) allows us to study the different effects of productivity, depreciation and discounting on the growth rate on consumption, along the time horizon. The effects of $\Omega, \Lambda$, and $\Delta$ are used throughout the-text to determine the growth rate of consumption through transition and in the steady state, as in figure 5. For any given damage function $D(P)$, the dynamic system of (17) and (18), with $\hat{C}$ from (19), along with the resource and climate dynamics (1), (3), and the transversality condition (16), are sufficient to completely characterize the decentralized economy. As shown in Bretschger and Karydas (2018), the dynamic system features a saddle-path stability, while it reaches a

\footnotetext{
${ }^{21}$ According to (19) below, we will impose a common restriction on the model's parameters such that $\lim _{t \rightarrow \infty} \alpha\left(B-D\left(P_{t}\right)\right)>\rho$; this ensures sufficient investment in capital accumulation for positive consumption growth, despite climate change damages.
} 
BGP when polluting resources get depleted, both asymptotically. The steady state values in our economy read:

$$
\begin{aligned}
& S_{\infty}=0, \\
& P_{\infty}=P_{\max }=P_{0}+\phi S_{0}, \\
& \hat{C}_{\infty}=g_{C}=\frac{1}{\sigma}\left(\alpha B-\alpha D\left(P_{\infty}\right)-\rho\right), \\
& u_{\infty}=(\sigma-1) g_{C}+\rho, \\
& \epsilon_{\infty}=(1 / B)\left((\sigma-1) g_{C}+\rho\right) .
\end{aligned}
$$

Our model of endogenous growth with nonlinear damages and CRRA utility does not allow for an analytical solution of the transition towards the steady state; we, therefore, rely on numerical simulations. We will solve the model by numerical differentiation using the Runge-Kutta method. Figure A1 of the online appendix shows graphically the outcome of the simulations for our baseline model. ${ }^{22}$

\subsection{Policy effects}

This section studies the effects of different climate policies on the evolution of the climate and the economic system. We will first study carbon taxation, where exogenously given taxes increase the consumer price of the polluting non-renewable resource. We will then examine the cases of using part of the available economic resources for abatement and adaptation, as well as the gradual decommissioning of the polluting resource stock.

\section{Carbon taxation}

Carbon taxes are the most widely studied policy instrument. This policy in the present macroeconomic context has been extensively analyzed, both for the social optimum and the decentralized equilibrium, in Bretschger and Karydas (2018), who focus on the lags in the climate system between the flow of emissions and damaging pollution. The following results can be retrieved as the limiting case of no-lags in the decentralized equilibrium of the aforementioned contribution.

Let $\tau$ represent given per-unit taxes on emissions $\phi R$, with $\phi>0$ the emissions intensity of the non-renewable resource. The first-order conditions for firms in the manufacturing sector read:

$$
(1-\alpha) \frac{Y_{t}}{R_{t}}=p_{R t}+\phi \tau_{t}, \quad \alpha \frac{Y_{t}}{\epsilon_{t} K_{t}}=p_{K t}, \quad p_{I t} B=p_{K t} .
$$

What changes is only the optimality condition for the employment of the non-renewable resource: its marginal cost is augmented by the effective tax rate $\phi \tau$. Let now $\psi \equiv$ $p_{R} /\left(p_{R}+\phi \tau\right)$ be the share of producer price $p_{R}$ in the consumer price of the nonrenewable resource, $p_{R}+\phi \tau$. Equation (18) continues to hold while the equivalent of

\footnotetext{
${ }^{22}$ The calibration of the baseline model closely follows Bretschger and Karydas (2018). Our initial time period is 2010, while for this numerical exercise we chose parameters on the damage function such that the growth rate of consumption starts at about 2 per cent p.a. converging to about 0.5 per cent p.a. in the long run. Specifically: $\sigma=1.8, \rho=0.015, \alpha=0.9, \delta_{0}=0.05, \delta_{1}=0.04, \delta_{2}=5 \times 10^{-9}, \eta=2.35, P_{0}=$ $830 G t C, S_{0}=6000 G t C, \phi=1, B=0.106$.
} 
(17) becomes:

$$
\hat{u}_{t}=u_{t}-(\sigma-1) \psi_{t} \hat{C}_{t}-\rho \psi_{t}-\left(1-\psi_{t}\right)\left(\hat{\tau}_{t}-\hat{C}_{t}\right)
$$

The dynamics of the tax are obviously of importance for the results. As is usually the outcome of such models with polluting non-renewable resources, the optimal tax is proportional to consumption when $\sigma=1$ (e.g., Golosov et al., 2014; Grimaud and Rouge, 2014), or it asymptotically becomes so in the long run for $\sigma \neq 1$ (e.g., Golosov et al., 2014; Bretschger and Karydas, 2018). Moreover, it is well established in the literature of the economics of non-renewable resources that any per unit tax that grows at a rate lower than the rate of interest delays resource extraction (e.g., Dasgupta and Heal, 1979). ${ }^{23}$ In light of the above, we will only study taxes that grow with consumption, i.e., $\hat{\tau}=\hat{C}$. With this conjecture, by log-differentiating the first equation of (25) we get:

$$
\hat{\psi}_{t}=\left(1-\psi_{t}\right)\left((\sigma-1) \hat{C}_{t}+\rho\right)
$$

From (14) and (15), $p_{R}$ grows at rate $r$, higher than $C$, and therefore $\tau$, implying that $\psi$ goes to unity as time goes to infinity. Following the same procedure as before with $\hat{\tau}=\hat{C}$, consumption growth reads:

$$
\begin{aligned}
\hat{C}_{t}= & \underbrace{\frac{\alpha B}{1+(\sigma-1)\left(\alpha+(1-\alpha) \psi_{t}\right)}}_{\Omega-\text { productivity }}-\underbrace{\frac{\left(\alpha+(1-\alpha) \psi_{t}\right) \rho}{1+(\sigma-1)\left(\alpha+(1-\alpha) \psi_{t}\right)}}_{\Delta \text {-discounting }} \\
& -\underbrace{\frac{\alpha D\left(P_{t}\right)}{1+(\sigma-1)\left(\alpha+(1-\alpha) \psi_{t}\right)}}_{\Lambda \text {-depreciation }},
\end{aligned}
$$

which asymptotically converges to (19) in the steady state for $\psi=1$. For a given damage function $D(P)$, and a carbon tax that grows with consumption, the dynamic system of (18), (26) and (27), with $\hat{C}$ from (28), along with the resource and climate dynamics (1), (3) and the transversality condition (16), are sufficient to completely characterize the evolution of decentralized economy.

The steady-state values of all variables (except $\psi$ ) are the same as before, i.e., equations (20)-(24). Hence carbon taxes affect the starting point and the transition of control variables $(\epsilon, u)$ but not the steady state of the economy. Resource taxation delays extraction and stretches the depletion of the resource stock to the future as can be seen in the left panel of figure A2 of the online appendix. During transition, pollution and damages are therefore always lower than in the baseline case, while consumption growth is always higher. Every variable converges to its long-run equilibrium, which is the same

\footnotetext{
${ }^{23}$ The case in which the tax increases with the resource rent would lead to carbon taxation having no effect on the dynamics of the economy (only a level effect). To see this, impose on the first equation of (25) a tax rate that grows as $p_{R}$. In this case the dynamics of the firms are the same as in the no-policy case. Since taxes are financed in a lump-sum manner by households, the problem of households stays unaltered. Therefore, this economy grows at the same rate as the benchmark economy. The last case in which the tax grows at a higher rate than the resource rent would affect the economy in the opposite way than the case we studied: resource extraction would accelerate, making consumption growth start from a lower point, while pollution and thus damages would accumulate faster. This would obviously be a bad environmental policy.
} 
as the baseline case. Finally, due to carbon taxation, the drag of resource extraction on growth is also lower in the beginning, which induces the growth rate of consumption to start from a higher level. ${ }^{24}$

In our setup, the social planner's solution would most closely resemble the above case of resource taxation. Resource extraction would be stretched further in time (i.e., a flatter extraction profile) and so would pollution accumulation. This would also induce a flatter growth profile towards the steady state (i.e., higher growth at all times in comparison to the no-policy case), where resources are being asymptotically depleted and pollution reaches its maximum value.

\section{Decommissioning of the resource stock}

A specific feature of models with non-renewable resources (abstracting from increasing extraction costs and backstop technologies) is that the optimal plans of resource owners lead to full exhaustion of the resource stock. As we have shown above, resource taxation simply shifts extraction to the future, without altering the total stock of carbon ultimately emitted into the atmosphere, i.e., $P_{\max }$ remains the same. However, a lower maximal pollution stock would exactly be needed for a climate policy, in line with our long-term targets, i.e., a global warming of $2^{\circ} \mathrm{C}$ - or even $1.5^{\circ} \mathrm{C}$ - by the end of the century. It is by now well understood among natural scientists and resource economists that some of the carbon assets must indeed be left in the ground to meet the internationally agreed temperature targets (Meinshausen et al., 2009; McGlade and Ekins, 2015).

This section examines decommissioning of the existing resource stock $S$ as a policy option. We will construct a simple thought experiment examining the problem from the side of the representative resource owner that faces a given expropriation policy each period with probability $1 .{ }^{25}$ When this policy is effective, it reduces the available stock of non-renewable resources by $N \in[0, S]$. We will further assume that the policy maker chooses the time path of policy $N_{t}$ which aims at decommissioning in total $\chi \in\left[0, S_{0}\right]$ units of polluting resources:

$$
\int_{0}^{\infty} N_{t} d t=\chi .
$$

According to the above, the resource stock dynamics now follow:

$$
\dot{S}_{t}=-R_{t}-N_{t}
$$

such that long-run pollution levels reach $P_{\text {max decom }}=P_{0}+\phi\left(S_{0}-\chi\right)$. Following the same procedure as in our baseline case, the appropriate dynamic budget constraint for

\footnotetext{
${ }^{24}$ To prove this, subtract (19) from (28) for $\sigma>1$ and $\psi_{t} \in(0,1)$ for a given $P_{0}>0$. Formal proofs of the rest can be found in Bretschger and Karydas (2018).

${ }^{25} \mathrm{~A}$ crucial assumption for an equilibrium to exist is that the policy is universal and effective simultaneously to every resource owner: let $x \in(0,1)$ be the constant expropriation rate effective from $t=0$, and let there be a continuum of infinitely lived households $i \in[0,1]$ owning the stock of capital and non-renewable resources. Following the same procedure as in the baseline case, capital and resource stocks of household $i$ evolve according to $\dot{K}_{i}=H_{i}-D(P) K_{i}$ and $\dot{S}_{i}=-R_{i}-x S_{i}$, while the dynamic budget constraint of household $i$ reads $\dot{W}_{i}=\theta_{i} W_{i}\left(\hat{p}_{R}-x\right)+\left(1-\theta_{i}\right) W_{i}\left(\hat{p}_{I}+B-D(P)\right)-C_{i}+T$. Maximizing utility w.r.t. $C_{i}$ and $\theta_{i}$, taking into account the dynamic budget constraint, leads to the usual Keynes-Ramsey rule (14) and the no-arbitrage equation (32), implying the existence of an equilibrium.
} 
the representative household reads:

$$
\dot{W}_{t}=\theta_{t} W_{t}\left(\hat{p}_{R t}-x_{t}\right)+\left(1-\theta_{t}\right) W_{t}\left(\hat{p}_{I t}+B-D\left(P_{t}\right)\right)-C_{t}+T_{t},
$$

with $\theta \equiv p_{R} S / W$, the share of the individual's resource wealth in the total assets, and $x \equiv$ $N / S$ the expropriation rate. The effect of policy $x$ reduces the profitability of the resource stock and alters the portfolio composition between stocks of capital. Accordingly, the no-arbitrage condition between assets, equation (15), now becomes:

$$
\hat{p}_{R t}-x_{t}=r_{t}=\hat{p}_{I t}+B-D\left(P_{t}\right) .
$$

The RHS of the equation that deals with the stock of physical capital remains the same, while the LHS changes by the $x$ term, the policy premium. The basic intuition is unchanged: adjusting for risk and depreciation, every asset should yield the same return. Accordingly, the resource owner should be compensated for the external political expropriation as proxied by parameter $x$, i.e. $\hat{p}_{R}=r+x$. The first-order conditions for firms, equations (9), and the Keynes-Ramsey rule (14), stay the same. Equation (30) with $u \equiv R / S$, yields $\hat{R}=\hat{u}-u-x$. Following the same procedure, the differential equations (17) and (18) remain the same, while consumption growth now becomes:

$$
\hat{C}_{t}=\underbrace{\frac{\alpha B}{\sigma}}_{\Omega \text {-productivity }}-\underbrace{\frac{\rho}{\sigma}}_{\Delta \text {-discounting }}-\underbrace{\frac{(1-\alpha) x_{t}}{\sigma}}_{\Pi \text {-policy }}-\underbrace{\frac{\alpha D\left(P_{t}\right)}{\sigma}}_{\Lambda \text {-depreciation }} .
$$

A given decommissioning policy path reduces the growth rate of consumption by the term $\Pi$ all along the transition and the steady state. A steeper price path of the resource with an effective policy does not lead to faster extraction as would be the case without the policy, because the total stock of available (polluting) resources is gradually reduced. Climate damages are lower during transition and in the steady state, since $P_{\text {max,decom }}=$ $P_{\text {max }}-\phi \chi$, with $P_{\max }=P_{0}+\phi S_{0}$. By comparing (19) with (33) as $t$ reaches infinity we see that as long as $x_{\infty}<\left(D\left(P_{\max }\right)-D\left(P_{\max , \text { decom }}\right)\right) \alpha /(1-\alpha)$, long-run economic development is promoted by the policy. The mechanism can be studied in figure A3 in the online appendix. ${ }^{26}$ Given $x_{\infty}$, steady states read: $P_{\infty}=P_{\text {max decom }}=P_{0}+\phi\left(S_{0}-\right.$ $\chi), \hat{C}_{\infty}=g_{C}=(1 / \sigma)\left(\alpha B-\alpha D\left(P_{\infty}\right)-\rho-(1-\alpha) x_{\infty}\right)$, with $S_{\infty}, u_{\infty}, \epsilon_{\infty}$ as in $(20)$, (23) and (24), respectively. Figure A3 of the online appendix shows graphically the dynamic development of the economy.

\section{Abatement}

This section deals with abatement as a policy option. We will formally study the case of carbon capture and sequestration (CCS) of figure $7(\mathrm{c}) .{ }^{27}$ We will assume that in order

\footnotetext{
${ }^{26}$ To construct figure A3 (online appendix), we assume that the dynamics of decommissioning are such that the expropriation rate $x=N / S$ reaches an asymptotic steady state $x_{\infty}$. According to (30) the dynamic equation of the expropriation rate is $\hat{x}=u+x-u_{\infty}-x_{\infty}$, while in the simulation we need to make sure that $x_{\infty}$ is chosen such that equation (29) is satisfied, i.e., $\int_{0}^{\infty} x_{t} S_{t} d t=\chi$. For the simulation we use a value of $\chi=0.25 \times S_{0}$.

${ }^{27}$ The case of adaptation of figure 7 (d) can be studied in a similar fashion; we abstract from this analysis to keep things concise.
} 
to proportionally reduce effective emissions each period by $\chi \in[0,1]$, the economy has to devote a part $X$ of the stock of physical capital, i.e.,

$$
\chi \phi R_{t}=\zeta X_{t} K_{t}
$$

with $\zeta>0$ a scaling parameter with appropriate units. Pollution stock dynamics now follow

$$
P_{t}=P_{0}+\phi(1-\chi)\left(S_{0}-S_{t}\right),
$$

while the growth rate of physical capital reads

$$
\hat{K}_{t}=B\left(1-\epsilon_{t}\right)-D\left(P_{t}\right)-X_{t}
$$

According to the above, abatement expenditure is an external action to the households, reducing the available stock of physical capital each period. Firms are facing the same demand curves, equations (9). The dynamic budget constraint of households changes to

$$
\dot{W}_{t}=\theta_{t} W_{t} \hat{p}_{R t}+\left(1-\theta_{t}\right) W_{t}\left(\hat{p}_{I t}+B-D\left(P_{t}\right)-X_{t}\right)-C_{t}+T_{t},
$$

which leads to the appropriate no-arbitrage condition between assets:

$$
\hat{p}_{R t}=r_{t}=\hat{p}_{I t}+B-D\left(P_{t}\right)-X_{t}
$$

In comparison to (32), due to abatement expenditure, households now expect higher net return from physical capital, i.e., $\hat{p}_{I}+B=r+D(P)+X$. Equations (17) and (18) still hold, while with the latter, (34) and (36), the dynamics of abatement expenditure rate $X$ reads:

$$
\hat{X}_{t}=X_{t}-(\sigma-1) \hat{C}_{t}-B\left(1-\epsilon_{t}\right)+D\left(P_{t}\right)-\rho .
$$

Finally, consumption growth becomes:

$$
\hat{C}_{t}=\underbrace{\frac{\alpha B}{\sigma}}_{\Omega \text {-productivity }}-\underbrace{\frac{\rho}{\sigma}}_{\Delta \text {-discounting }}-\underbrace{\frac{\alpha X_{t}}{\sigma}}_{\Pi \text {-policy }}-\underbrace{\frac{\alpha D\left(P_{t}\right)}{\sigma}}_{\Lambda \text {-depreciation }} .
$$

Given policy $\chi$, initial conditions $S_{0}, P_{0}$, steady states $\lim _{t \rightarrow \infty} X_{t}=0, \lim _{t \rightarrow \infty} \epsilon_{t}=$ $\epsilon_{\infty}, \lim _{t \rightarrow \infty} u_{t}=u_{\infty}$, and equations (35), (17), (18), (39) and (40) are sufficient to completely characterize the dynamic evolution of the economy at hand. Just as in the case of decommissioning, economic growth starts from a lower level due to policy, reaching however a much higher steady state due to lower pollution and damages. The steady states are: $X_{\infty}=0, P_{\infty}=P_{\text {max } \text {,abate }}=P_{0}+\phi(1-\chi) S_{0}, \hat{C}_{\infty}=g_{C}=$ $(1 / \sigma)\left(\alpha B-\alpha D\left(P_{\infty}\right)-\rho\right)$, with $S_{\infty}, u_{\infty}, \epsilon_{\infty}$ as before. Figure A4 in the online appendix graphically presents the results.

\section{Comparing with the literature}

The strength of our BCE model is that, besides its simplicity, it can incorporate relevant features on the interconnection between climate change and macroeconomics such as polluting non-renewable resources as a productive input, pollution-induced damages to 
physical capital, and perpetual growth, based on the endogenous decisions of households between investment and consumption. It is constructive at this point to compare our model with models that have drawn attention in the literature, namely, the DICE model (Nordhaus, 2017) and the model of Golosov et al. (2014).

The DICE model - short for Dynamic Integrated model of Climate and the Economy - pioneered the literature of climate economics in the 1970s and has been extensively used to model the macroeconomic implications of climate change ever since. At its core lies a Ramsey growth engine that allows for a social planner's solution of optimal warming but not for endogenous growth. Market structure and generic climate policies, like the ones presented in the previous section, are not specified. Production inputs in the DICE model are physical capital and labor. ${ }^{28}$ Economic output causes manmade climate change which in turn affects total factor productivity but not capital stock. Due to the complex climate dynamics used, the results attained from the DICE model come in the form of numerical simulations. Our analysis is positive and not normative; it shows the different policy effects with the inclusion and intuitive study of several relevant but possibly suboptimal policies in decentralized equilibrium. We include polluting depletable resources, endogenous growth, different forms of damage functions, and the latest development in the field of environmental science, in particular the linearity of climate change in emissions. Also, our setup allows for the derivation of analytical solutions, depending on the assumptions on preferences and damages.

The contribution of Golosov et al. (2014) also focuses on analytical solutions. Using a Ramsey-type model like in DICE, it includes polluting non-renewable resources as a productive input and adopts climate dynamics which are less complex than DICE. The authors solve for the decentralized equilibrium and the social optimum. The model assumes full capital depreciation. Capital is thus no longer treated as a stock variable in the model; it is not harmed by climate change as it is in our approach. Under these conditions, three specific model assumptions allow for a closed-form solution for the social cost of carbon (SCC): (i) the logarithmic specification of the utility function, (ii) the resulting constant savings rate in every time period, and (iii) the specification of the damage function which approximates the DICE climate damages with an exponential damage function in effective output. From this the authors derive an optimal carbon tax per unit of polluting resources which is linear in consumption.

As an illustration of this simplification procedure, take the example of the Ramseytype economy of Golosov et al. (2014), but in continuous time, with pollution damages $G(P)$ in aggregate production, and with the same climate dynamics as in our model (equation (2)). ${ }^{29}$ Effective output in each period reads $(1-G(P)) Y$, with $Y$ denoting gross production and the numeraire good. In this economy, the social cost of carbon, $\Lambda^{G}$, is given by the following pricing equation: $\Lambda_{t}^{G}=\int_{t}^{\infty} \underbrace{\left(m_{v} / m_{t}\right)}_{I} \times \underbrace{\phi G^{\prime}\left(P_{v}\right) Y_{v}}_{I I} d v$,

\footnotetext{
${ }^{28}$ Although the model abstracts from non-renewable resources as a direct input in production, its production includes a marginal abatement cost which implicitly defines the marginal productivity of fossil fuels. Moreover, by placing an upper bound on the available stock of carbon, the DICE model also allows for Hotelling rents to be made. We thank an anonymous referee for this comment.

${ }^{29}$ In Golosov et al. (2014), the authors consider $P$ to be atmospheric concentration instead of cumulative emissions (or temperature anomaly) and approximate the damages of the DICE model with an exponential function of the sort that we presented above. In fact, by doing so they combine the typical damage function with temperature anomaly (or cumulative emissions) as an argument, with another function that maps carbon concentration into temperature anomaly.
} 
with $m_{t} \equiv U^{\prime}\left(C_{t}\right) e^{-\rho t}$. The above equation measures the discounted stream of marginal damages from date $t$ and forever. The first term in the integral (I), i.e., the ratio $U^{\prime}\left(C_{v}\right) e^{-\rho v} / U^{\prime}\left(C_{t}\right) e^{-\rho t}$, is the marginal rate of substitution between consuming today, or in a subsequent period $v>t$, and is responsible for discounting. The second term (II) represents the marginal damage on final output from extracting and burning an additional unit of polluting resources in period $v$, and can be thought of as the current negative dividend of emissions in a given period $v$. Golosov et al. (2014) specify damages in an exponential form of the sort $G(P)=1-e^{-\gamma\left(P-P_{0}\right)}$, implying that $G^{\prime}(P) Y=$ $\gamma(1-G(P)) Y$. With this conjecture, the SCC reads:

$$
\Lambda_{t}^{G}=\phi \gamma C_{t} \int_{t}^{\infty} \frac{\left(1-G\left(P_{v}\right)\right) Y_{v}}{C_{v}}\left(\frac{C_{t}}{C_{v}}\right)^{\sigma-1} e^{-\rho(v-t)} d v .
$$

The last equation readily allows for a closed form solution and the linearity of the SCC in consumption (or output) if two conditions are met: first, $\sigma=1$, i.e., the utility is logarithmic; and second, the savings rate is constant, leading to a constant ratio $(1-G(P)) Y / C$. The last condition is satisfied in the discrete time framework of Golosov et al. (2014) when capital depreciates fully each period.

In contrast to both aforementioned contributions, we incorporate damages directly into capital accumulation (see equation (7)). Our view is that adverse climate-related events, caused by man-made climate change, destroy stocks of capital such as buildings, equipment, crops, roads, and public infrastructure every year. Since part of the, otherwise productive, available economic resources have to be allocated to fixing damages, this puts a natural drag on economic development. In our economy, marginal damages on the value of the stock of capital from an additional unit of emissions in time $v$, read $\phi D^{\prime}\left(P_{v}\right) p_{K v} K v$. Our pricing equation for the SCC, $\Lambda^{B C E}$, with $p_{K v} K_{v}=\alpha / B C_{v} / \epsilon_{v}$ from the second equation of (10), implies:

$$
\Lambda_{t}^{B C E}=\frac{\phi \alpha}{B \epsilon_{\infty}} C_{t} \int_{t}^{\infty} D^{\prime}\left(P_{v}\right)\left(\frac{\epsilon_{\infty}}{\epsilon_{v}}\right)\left(\frac{C_{t}}{C_{v}}\right)^{\sigma-1} e^{-\rho(v-t)} d v,
$$

where $\epsilon$, i.e., capital allocation share between consumption and investment, plays the role of the savings rate in our endogenous growth setting, and $\epsilon_{\infty}$ as in (24). ${ }^{30}$ According to (42), the linearity of the SCC in consumption is warranted with $\sigma=1$, and when damages in capital accumulation are linear in pollution, i.e., $D^{\prime}(P)$ a constant. ${ }^{31}$

\section{Conclusions}

The paper was motivated by the need for a flexible and intuitive climate economics framework including the core elements of the economy and the climate system. As a response to the gap in the literature, we have developed the basic climate economic (BCE) model which features resource extraction, pollution accumulation, climate damage functions and endogenous growth. In the first part, we have shown graphically how the different functional forms and climate policies have an impact on long-run development. The focus was to demonstrate that the setup is versatile and intuitive, allowing

\footnotetext{
${ }^{30}$ With $C+p_{I} I$ measuring GDP, the savings rate reads $s \equiv p_{I} I /\left(C+p_{I} I\right)$. Using the second and third equations in (9), with $I=B(1-\epsilon) K$, we get that $s=1 /(1+(1 / \alpha) \epsilon /(1-\epsilon))$. As $\epsilon \rightarrow 0, s \rightarrow 1$, and vice versa.

${ }^{31}$ As follows from equations (16) and (18), for $\sigma=1, \epsilon=\rho / B$ in every time period.
} 
for broad use in education and communication. In the second part, we have provided the analytical foundation for all the functional forms and a derivation of the analytical results. A final contribution concerned the comparison of the BCE model to existing climate models.

The model could be extended to include more elements suuch as resource extraction costs, resource discoveries, more specific damage functions, technical innovations, education or more sectors of the economy. Also, the range of considered policies could be enlarged. As there are big regional differences in economic performance and climate vulnerability, a regionalized version could also be considered. These issues are left for future research.

Supplementary Material. The supplementary material for this article can be found at https://doi.org/10. 1017/S1355770X19000184

Acknowledgements. This study has benefited greatly by the comments and suggestions of the editors and three anonymous referees. Moreover we would like to thank Sjak Smulders, Aimilia Pattakou, and the participants of the FSR Climate Annual Conference 2017 for their valuable contributions.

\section{References}

Bretschger L (2017) Climate policy and economic growth. Resource and Energy Economics 49, 1-15.

Bretschger L and Karydas C (2018) Optimum growth and carbon policies with lags in the climate system. Environmental and Resource Economics 70, 781-806.

Bretschger L and Pattakou A (2019) As bad as it gets: how climate damage functions affect growth and the social cost of carbon. Environmental and Resource Economics 72, 5-26.

Bretschger L and Smulders S (2012) Sustainability and substitution of exhaustible natural resources: how structural change affects long-term R \& D-investments. Journal of Economic Dynamics and Control 36, 536-549.

Bretschger L and Vinogradova A (2018) Escaping Damocles' sword: endogenous climate shocks in a growing economy. Technical report, Economics Working Paper Series 18/291, ETH Zurich.

Brock W and Xepapadeas A (2017) Climate change policy under polar amplification. European Economic Review 94, 263-282.

Dasgupta P and Heal G (1974) The optimal depletion of exhaustible resources. The Review of Economic Studies 41, 3-28.

Dasgupta P and Heal G (1979) Economic Theory and Exhaustible Resources. Cambridge, UK: Cambridge University Press.

Dietz S and Stern N (2015) Endogenous growth, convexity of damage and climate risk: how Nordhaus' framework supports deep cuts in carbon emissions. The Economic Journal 125, 574-620.

Dietz S and Venmans F (2017) Cumulative carbon emissions and economic policy: in search of general principles. Technical report, London School of Economics.

Farmer JD, Hepburn C, Mealy P and Teytelboym A (2015) A third wave in the economics of climate change. Environmental and Resource Economics 62, 329-357.

Felbermayr $\mathbf{G}$ and Licandro $\mathbf{O}$ (2005) The underestimated virtues of the two-sector AK model. Contributions to macroeconomics. The B.E. Journal in Macroeconomics 5(1).

Gaudet G (2007) Natural resource economics under the rule of Hotelling. The Canadian Journal of Economics 40, 1033-1059.

Gaudet G and Lasserre P (2015) The taxation of nonrenewable natural resources. In Halvorsen R and Layton D (eds), Handbook on the Economics of Natural Resources. Cheltenham, UK: Edward Elgar Publishing, pp. 66-96.

Gerlagh R and Liski M (2017) Consistent climate policies. Journal of the European Economic Association $16,1-44$.

Gerlagh R and Liski M (2018) Carbon prices for the next hundred years. The Economic Journal 128, $728-757$. 
Golosov M, Hassler J, Krusell P and Tsyvinski A (2014) Optimal taxes on fossil fuel in general equilibrium. Econometrica 82, 41-88.

Grimaud A and Rouge L (2014) Carbon sequestration, economic policies and growth. Resource and Energy Economics 36, 307-331.

Hassler J, Krusell P and Olovsson C (2015) Energy-saving technical change. Discussion papers 1529, Centre for Macroeconomics (CFM).

Hassler J, Krusell P and Smith A (2016) Environmental macroeconomics. In Taylor J and Uhlig H (eds), Handbook of Macroeconomics, Volume, 2B, Elsevier B.V., pp. 1893-2008.

Hotelling H (1931) The economics of exhaustible resources. Journal of Political Economy 39, 137-175.

IPCC (2013) Climate Change 2013: The Physical Science Basis. WG I Contribution to the IPCC 5, Summary for policy makers, Cambridge, UK and New York, NY: Cambridge University Press.

Jones CI (1995) Time series tests of endogenous growth models. The Quarterly Journal of Economics 110, 495-525.

Lemoine D and Traeger C (2014) Watch your step: optimal policy in a tipping climate. American Economic Journal: Economic Policy 6, 137-66.

Matthews HD and Caldeira K (2008) Stabilizing climate requires near-zero emissions. Geophysical Research Letters 35, L04705.

Matthews HD, Gillett N, Stott P and Zickfeld K (2009) The proportionality of global warming to cumulative carbon emissions. Nature 549, 829-832.

McGlade C and Ekins P (2015) The geographical distribution of fossil fuels unused when limiting global warming to $2^{\circ} \mathrm{C}$. Nature $517,187-190$.

Meinshausen M, Meinshausen N, Hare W, Raper SCB, Frieler K, Knutti R, Frame DJ and Allen MR (2009) Greenhouse-gas emission targets for limiting global warming to $2^{\circ} \mathrm{C}$. Nature 458, 1158-1163.

Nordhaus WD (2017) Revisiting the social cost of carbon. Proceedings of the National Academy of Sciences 114, 1518-1523.

Papageorgiou C, Saam M and Schulte P (2017) Substitution between clean and dirty energy inputs: a macroeconomic perspective. The Review of Economics and Statistics 99, 281-290.

Pigou AC (1920) The Economics of Welfare. London: Macmillan.

Pindyck RS (2013) Climate change policy: what do the models tell us? Journal of Economic Literature 51, 860-872.

Rebelo S (1991) Long-run policy analysis and long-run growth. Journal of Political Economy 99, 500-521.

Stern N (2007) The Economics of Climate Change: The Stern Review. Cambridge, UK: Cambridge University Press.

Stern N (2016) Current climate models are grossly misleading. Nature 530, 407-409.

van den Bijgaart I, Gerlagh R and Liski M (2016) A simple formula for the social cost of carbon. Journal of Environmental Economics and Management 77, 75-94.

van der Ploeg F and Withagen C (2012) Is there really a green paradox? Journal of Environmental Economics and Management 64, 342-363.

van der Werf E (2008) Production functions for climate policy modeling: an empirical analysis. Energy Economics 30, 2964-2979.

Weitzman ML (2010) Risk-adjusted gamma discounting. Journal of Environmental Economics and Management $60,1-13$.

Cite this article: Bretschger L, Karydas C (2019). Economics of climate change: introducing the Basic Climate Economic (BCE) model. Environment and Development Economics 24, 560-582. https://doi.org/10. $1017 /$ S1355770X19000184 